\title{
Using Correlative Light and Electron Microscopy (CLEM) to Understand Ultrastructural Changes Induced by Salmonella typhimurium Infection in a Calf Ileum Loop Model
}

Sophia Antonioli-Schmit ${ }^{1}$, Audrey Chong ${ }^{2}$, Bryan T Hansen ${ }^{3}$, Mikayla Pulliam ${ }^{3}$, Vinod Nair ${ }^{3 *}$

1. Montana State University, Microbiology, Bozeman, Montana, USA.

2. NIH/NIAID/ RML/Laboratory of Bacteriology, Hamilton, Montana, USA.

3. NIH/NIAID/RML/RTB/EM Unit, Hamilton, Montana, USA.

* Corresponding author: nairv@niaid.nih.gov

Correlative light and electron microscopy (CLEM) is a powerful technique to study rare, dynamic or transient events. Using CLEM, we can identify events of interest efficiently with classical light microscopy techniques while gaining better ultra-structural details of the same region using transmission electron microscopy (TEM). Performing CLEM on cultured cells grown on coverslips has become a routine technique. However, locating a region of interest in tissue still has some challenges [1]. We have attempted to demystify this technique by studying in vivo Salmonella infection using a calf ligated ileum loop model [2]. Initial experiments involved testing the effect of various fixation conditions on the ultrastructure of ileum loops infected with high dose of bacteria (Fig. 1A). Hematoxylin and eosin (H\&E) stained histology slides revealed villous blunting, a pathology induced by Salmonella infection (Fig. 1B). By TEM, tissue biopsies fixed in $2.5 \%$ glutaraldehyde with $2.5 \%$ paraformaldehyde in $0.1 \mathrm{M}$ sodium cacodylate buffer revealed well preserved bacteria contained in a membrane bound vacuole (Fig.1C).

Genes encoded by Salmonella pathogenicity island 1 (SPI1) play an important role in its ability to invade the non-phagocytic cells of the gut. Detecting bacteria by TEM to investigate the ultra-structural changes resulting from early infection and invasion using biologically relevant doses of wild type (WT) and SPI1 mutant strains of Salmonella has been challenging. Preliminary CLEM analysis was performed on fluorescently labeled biopsy pieces that were fixed in $10 \%$ formalin, floated in sucrose and sectioned using a cryostat (Fig 2A.). Slides with sections showing Salmonella infected tissue were fixed with $2.5 \%$ glutaraldehyde and processed for TEM analysis. Although subsequent sections collected on the cryostat processed for TEM analysis allowed for successful localization of infected epithelial cells in the tissue, this processing had undesirable effects on ultrastructural morphology (Fig.2B).

Alternate CLEM techniques like vibratome sectioning of fixed tissue pieces screened by light microscopy and processed using conventional EM fixatives is being currently investigated. This technique would overcome the shortcomings of CLEM performed on cryostat sections offering a better understanding of the science of infection and invasion of this pathogenic bacteria [3].

\section{References:}

[1] C. Loussert, C. L. Forestier and B. M. Humbel in "Methods in Cell Biology", Editor(s) T. MüllerReichert and P. Verkade, (Academic Press), 111 (2012), p. 59-73.

[2] R. C. Laughlin et al. MBio 5 (2014), p. e00946-13.

[3] The authors would like to acknowledge Olivia Steele-Mortimer, Laszlo Kari, Elizabeth R. Fischer, Forrest Hoyt and Leigh Knodler for their valuable insight and contribution to this work. This study was supported by Division of Intramural Research, NIAID, NIH. 

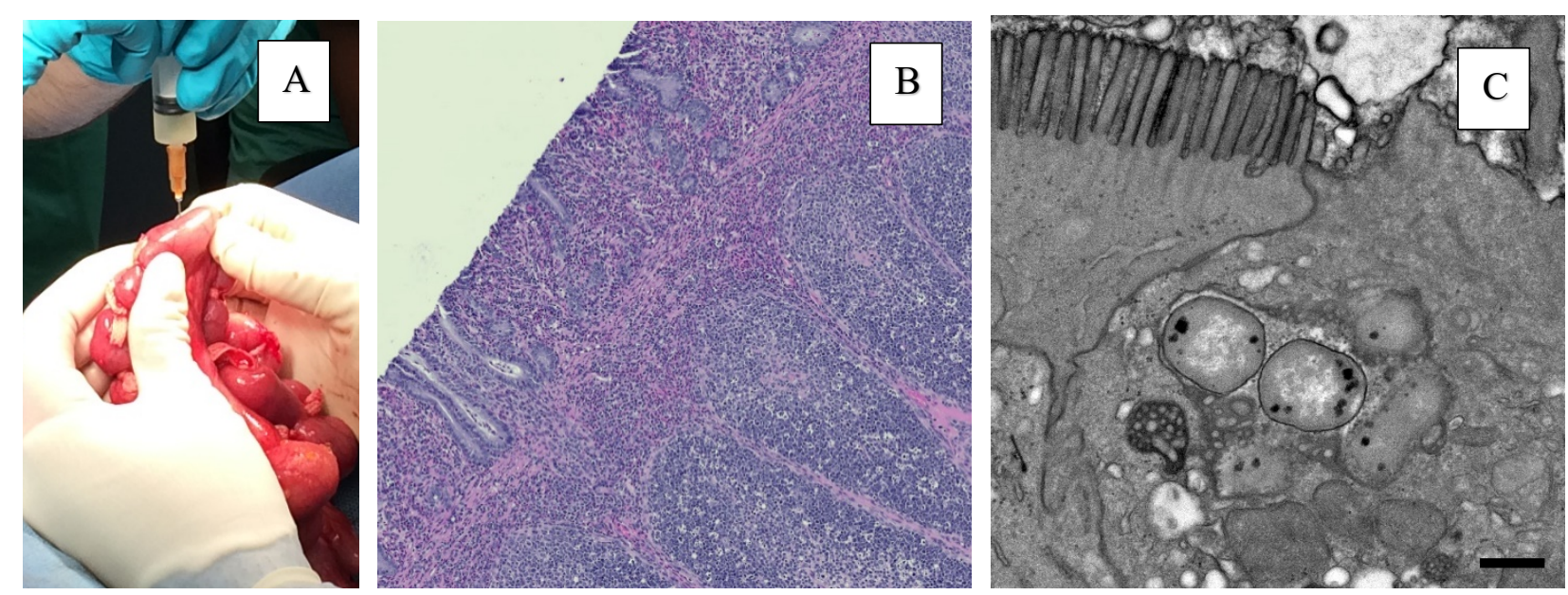

Figure 1. (A) Infecting ligated ileum loops with Salmonella. (B )H\&E stained biopsy showing villous blunting (C) TEM image showing Salmonella in membrane bound vacuoles. Scale bar $=500 \mathrm{~nm}$.
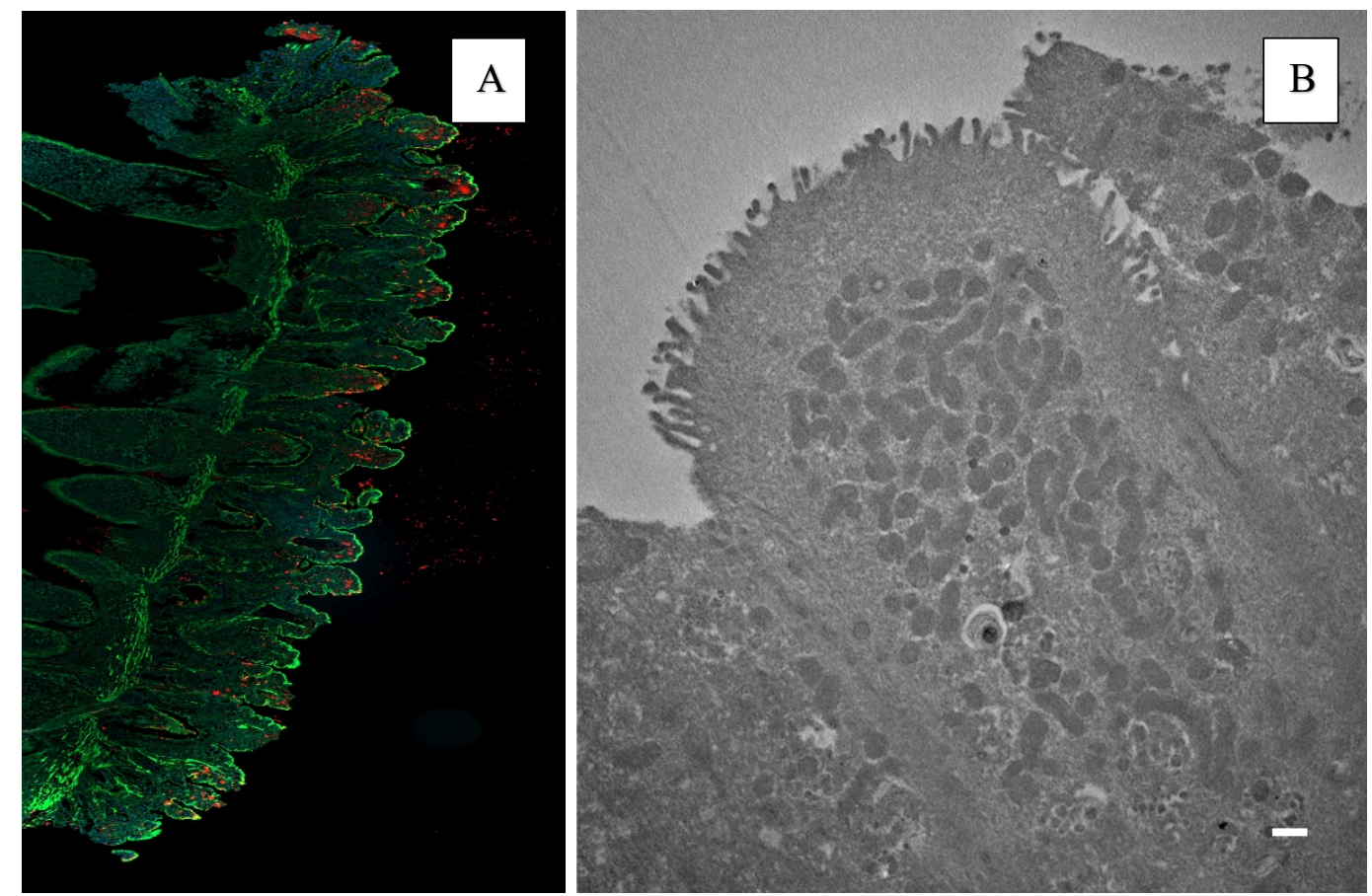

Figure 2. (A) Immunofluorescence image of cryostat sectioned tissue showing Salmonella in red and actin in green. (B) TEM images of the section showing sub optimal ultrastructure. Scale bar $=500 \mathrm{~nm}$. 